Artículos Científicos

\title{
El ente garante de la regulación jurídica del cannabis en México
}

The guarantor entity of legal regulation of cannabis in Mexico

O garante da regulamentação legal da cannabis no México

Alejandro Sánchez Sánchez

Universidad Autónoma de Baja California, México alexsasacc@uabc.edu.mx https://orcid.org/0000-0002-4527-7308

Gloria Aurora De Las Fuentes Lacavex Universidad Autónoma de Baja California, México gloriaaurora@uabc.edu.mx https://orcid.org/0000-0001-8936-4959

Luis Carlos Castro Vizcarra Universidad Autónoma de Baja California, México luiscarloscastro@uabc.edu.mx https://orcid.org/0000-0002-3272-2388

Alejandro Moctezuma Hernández Universidad Autónoma de Baja California, México almoctezuma@uabc.edu.mx https://orcid.org/0000-0001-8832-2669 


\section{Resumen}

Este análisis aborda el sistema jurídico del cannabis en México estableciendo la naturaleza jurídica del ente garante y las facultades del Instituto Mexicano de Regulación y Control del Cannabis (IMRCC). Se compara la propuesta de política en México con la Ley de Reglamentación e Impuestos de Cannabis del estado de Illinois, Estados Unidos. Lo anterior con el fin de relacionar, describir y explicar la problemática del fenómeno social del consumo con una política prohibitiva absoluta contradictoria al derecho fundamental al libre desarrollo de la personalidad. Como parte de las conclusiones se propone una serie de medidas que deberán ser puestas en práctica para cristalizar el derecho fundamental al libre desarrollo de la personalidad y dar solución positiva, legal y constitucional al fenómeno social analizado.

Palabras clave: cannabis, ente público, México, regulación.

\section{Abstract}

This analysis addresses the legal system of cannabis in Mexico establishing the legal nature of the guarantor entity and the powers of the Instituto Mexicano de Regulación y Control del Cannabis (IMRCC). It compares the policy proposal in Mexico with the Cannabis Regulation and Tax Act of the state of Illinois of the United States of America. This in order to relate, describe and explain the problems of the social phenomenon of consumption with an absolute prohibitive policy contradictory to the fundamental right to the free development of personality. As part of the conclusions, a series of measures are proposed that must be put into practice to crystallize the fundamental right to free development of personality and provide a positive, legal and constitutional solution to the social phenomenon analyzed.

Keywords: cannabis, public entity, Mexico, regulation. 


\section{Resumo}

Esta análise aborda o Sistema jurídico da cannabis no México, estabelecendo a natureza jurídica da entidade garante e os poderes do Instituto Mexicano de Regulação e Controle da Cannabis (IMRCC). A proposta de política no México é comparada com a Lei de Regulamentação e Impostos de Cannabis do estado de Illinois, Estados Unidos. O exposto, a fim de relacionar, descrever e explicar os problemas do fenômeno social do consumo com uma política proibitiva absoluta, contraditória ao direito fundamental, o livre desenvolvimento da personalidade. Como parte das conclusões, propõe-se uma série de medidas que devem ser postas em prática para cristalizar o direito fundamental ao livre desenvolvimento da personalidade e fornecer uma solução positiva, legal e constitucional para o fenômeno social analisado.

Palavras-chave: cannabis, entidade pública, México, regulamentação.

Fecha Recepción: Mayo 2019

Fecha Aceptación: Diciembre 2019

\section{Introducción}

Más allá de la propuesta de ley que propone la creación del Instituto Mexicano de Regulación y Control del Cannabis (IMRCC) no existen antecedentes en México de un organismo con tales fines, por lo que, al aprobarse esta, se iniciaría un camino anteriormente no transitado. Dicha propuesta, con la que se pretende el control sanitario del cannabis para uso personal, científico y comercial, se presentó el día 18 de noviembre de 2018.

El presente análisis aborda un fenómeno social de salud pública desde la faceta de las ciencias jurídicas, y da respuesta a las siguientes interrogantes: ¿cuál es la naturaleza jurídica del ente garante de la regulación y control del cannabis?, ¿cuáles son sus facultades? y ¿cuáles son sus similitudes y diferencias con el de otro país? Así pues, se establecen como objetivos determinar la naturaleza jurídica del ente garante de la regulación y control del cannabis y establecer sus facultades correlacionándolo con el de otra nación. Para ello, se utiliza un enfoque cualitativo y cuantitativo, con un alcance indagatorio, descriptivo, correlacional y explicativo, utilizando como hilo conductor de la investigación al organismo que será responsable de regular y controlar el cannabis en México. 
Se establece como hipótesis la siguiente:

H1. La creación de un ente garante que regule jurídicamente el cannabis en México coadyuvará e impactará positivamente al objetivo de establecer una estructura jurídica constitucional y legal que sirva como marco normativo para el desarrollo de las actividades relacionadas con este fenómeno social en nuestro país.

El desarrollo y las conclusiones a las que se arriben partirán de premisas estipuladas en la Constitución Política de los Estados Unidos Mexicanos, la jurisprudencia, las leyes secundarias, la doctrina, los datos estadísticos sobre el fenómeno en estudio y el comparativo binacional; todo ello, por supuesto, de las deducciones e inducciones de los autores. Al ser un tema innovador en México, en este estudio se pretende delimitar y, a la vez, invitar a indagar al respecto, con la finalidad de que se logre una política que cristalice el derecho fundamental al libre desarrollo de la personalidad, contando con la existencia del ente garante de la regulación jurídica del cannabis en México.

\section{Naturaleza jurídica del IMRCC}

La administración pública federal en los Estados Unidos Mexicanos se divide, para su ejercicio, en centralizada y paraestatal. La centralizada está integrada por la Oficina de la Presidencia de la República, las secretarías de Estado, la Consejería Jurídica del Ejecutivo Federal y los órganos reguladores coordinados. La paraestatal está conformada por los organismos descentralizados, las empresas de participación estatal, las instituciones nacionales de crédito, las organizaciones auxiliares nacionales de crédito, las instituciones nacionales de seguros y de fianzas y los fideicomisos.

El IMRCC será un órgano descentralizado de la administración pública federal a cargo de la Secretaría de Salud. Su misión será regular, reglamentar, monitorear, sancionar y evaluar el sistema de regulación del cannabis. Y su fuente constitucional como ente público se encuentra en la Constitución Política de los Estados Unidos Mexicanos (Cámara de Diputados del H. Congreso de la Unión, 1917). Allí se establece lo siguiente:

La Administración Pública Federal será centralizada y paraestatal conforme a la Ley Orgánica que expida el Congreso, que distribuirá los negocios del orden administrativo de la Federación que estarán a cargo de las Secretarías de Estado y definirá las bases generales de creación de las entidades paraestatales 
Revista Iberoamericana

de las Ciencias Sociales y

Humanísticas

ISSN: $2395-7972$

y la intervención del Ejecutivo Federal en su operación (Cámara de Diputados del H. Congreso de la Unión, 1917, art. 90).

Del numeral citado se origina la Ley Orgánica de la Administración Pública Federal (Diario Oficial de la Federación [DOF], 29 de diciembre de 1976). En sus artículos 3 y 17 se encuentran las bases legales para la existencia de los organismos descentralizados como lo es el IMRCC. El primero de ellos indica: "El Poder Ejecutivo de la Unión, se auxiliará en los términos de las disposiciones legales correspondientes, de las siguientes entidades de la administración pública paraestatal, organismos descentralizados” (DOF, 29 de diciembre de 1976). El segundo establece:

Para la más eficaz atención y eficiente despacho de los asuntos de su competencia, las Secretarías de Estado podrán contar con órganos administrativos desconcentrados que les estarán jerárquicamente subordinados y tendrán facultades específicas para resolver sobre la materia y dentro del ámbito territorial que se determine en cada caso, de conformidad con las disposiciones legales aplicables (DOF, 29 de diciembre de 1976, art. 17).

Serra (2013) sostiene que la administración pública paraestatal está integrada por el conjunto de instituciones, organismos, empresas de economía mixta, patrimonios públicos, que, por disposición de la ley, colaboran en la relación de los fines del Estado, sin formar parte de la administración pública centralizada, con la cual mantienen estrictas relaciones de control y vigilancia a cargo de aquella, y dividida en sectores para tal efecto.

La Segunda Sala de la Suprema Corte de Justicia de la Nación (2013) define la naturaleza jurídica de los organismos descentralizados (incluyendo por tanto al IMRCC) mediante la tesis que se cita a continuación:

Esta Suprema Corte de Justicia de la Nación ha determinado que los organismos descentralizados, ubicados dentro de la administración pública paraestatal, fuera de la administración pública centralizada, son entidades creadas por ley o decreto del Congreso de la Unión o por decreto del Ejecutivo Federal, con personalidad jurídica y patrimonio propios. Asimismo, ha sostenido que dichas entidades paraestatales, para el cabal cumplimiento de su objeto y de los objetivos y metas señalados en sus programas, gozan de autonomía de gestión, además cuentan con una administración a cargo de un 
Revista Iberoamericana

de las Ciencias Sociales y

Humanísticas

ISSN: $2395-7972$

órgano de gobierno, el cual debe expedir el estatuto orgánico, y de un director general, quien tiene la representación legal del organismo, siendo así que, como entidad con personalidad jurídica propia, es diversa a la atinente al "Presidente", "Presidente de la República", "Ejecutivo Federal" o "Poder Ejecutivo de la Unión", que si bien tiene a su cargo el desarrollo de la función administrativa del Estado mexicano en el orden federal, interviniendo junto con sus dependencias, estableciendo políticas para el logro de los objetivos y prioridades de la planeación nacional del desarrollo y objetivos, lo cierto es que sus relaciones con los organismos descentralizados están sujetas a lo que establece la Ley Federal de las Entidades Paraestatales (reglamentaria del artículo 90 de la Constitución Política de los Estados Unidos Mexicanos) y a sus disposiciones reglamentarias y específicas de la materia. Por consiguiente, dada su autonomía jerárquica, los organismos descentralizados no están subordinados al Presidente de la República, pues este ejerce solo un control de manera mediata e indirecta, en tanto que la relación de jerarquía directa en la administración pública paraestatal no existe con el Poder Ejecutivo (párr. $1)$.

La Ley Federal de las Entidades Paraestatales (DOF, 26 de enero de 1990) tiene por objeto regular la organización, funcionamiento y control de las entidades paraestatales de la administración pública federal. Asimismo, indica que las relaciones del Ejecutivo Federal, o de sus dependencias, con las entidades paraestatales, en cuanto unidades auxiliares de la administración pública federal, se sujetarán, en primer término, a lo establecido en esta misma ley, y sus disposiciones reglamentarias y, solo en lo no previsto, a otras disposiciones según la materia que corresponda.

De Pina García, de Pina Vara y de Pina (2012), en su Diccionario de Derecho, definen la naturaleza jurídica de los organismos descentralizados.:

Son consideradas como tales las instituciones creadas por disposición del Congreso de la Unión, o en su caso por el Ejecutivo Federal, con personalidad jurídica y patrimonio propios, cualquiera que sea la estructura legal que adopten; son organismos descentralizados las personas jurídicas creadas conforme a lo dispuesto por la Ley Orgánica de la Administración Pública Federal y cuyo objeto sea: 1. La realización de actividades correspondientes a 
Revista Iberoamericana

de las Ciencias Sociales y

Humanísticas

ISSN: $2395-7972$

las áreas estratégicas o prioritarias; 2. La prestación de un servicio público o social; y, la obtención o aplicación de recursos para fines de asistencia o seguridad social (p. 391).

Hamdan (2016), por su parte, define a los organismos descentralizados estableciendo que en la organización interna de las Secretarías de Estado tienen áreas de decisión, supervisión, evaluación, administrativas, operativas; tienen personalidad jurídica y patrimonio propio. El Ejecutivo Federal, en ejercicio de sus facultades para crear órganos subordinados a las Secretarías de Estado, distribuye la competencia que a cada órgano le compete y le corresponde. Hamdan (2016) afirma que técnicamente la palabra descentralización apunta a que algo sale del centro hacia la periferia relajando su vinculación con la administración centralizada. Además, sostiene que los organismos descentralizados son creados por ley del Congreso de la Unión, a pesar de que la Ley Orgánica de la Administración Pública Federal señala que pueden ser creadas por el Poder Ejecutivo Federal.

De las premisas anteriores, se deduce que el ente garante que se titulará IMRCC pertenecerá a la Secretaría de Salud; sin embargo, se observa una contradicción en virtud de que la iniciativa de ley propone que pertenezca a la Secretaría de Gobernación, por la gran trascendencia e impacto que tendrá en los sectores que de hecho atienden este tema. Se considera que el ente debe ser integrado por tres dependencias: la primera para garantizar las variables de salud, la segunda, la Comisión Federal para la Protección contra Riesgos Sanitarios (Cofepris), para establecer las políticas administrativas para la regulación del psicotrópico y la tercera, la Secretaría de Gobernación, para las cuestiones de seguridad.

\section{Las facultades del IMRCC}

Por la trascendencia, importancia, riesgo e impacto social que este fenómeno tiene en la sociedad mexicana, el IMRCC requerirá que se le dote de facultades fortalecidas en la ley y basadas en los principios constitucionales, para poder operar y hacer realidad el derecho fundamental que debe cristalizar. Sánchez Cordero y Monreal Ávila (jueves 8 de noviembre de 2018) sostienen que entre esas deberá contar con una facultad sancionadora, de capacitación, de proveedor, registral, de cesión de permisos, de investigación científica, de determinación de tributos, establecer las destrezas para el transporte, los puntos de venta, las 


\section{Revista Iberoamericana \\ de las Ciencias Sociales y Humanísticas}

ISSN: 2395 - 7972

características de embalaje, así como autorizar el funcionamiento de cada una de las fases que integrarán el sistema.

En su iniciativa con proyecto de decreto ambos senadores sostienen que la política prohibicionista que México adoptó ha generado dos consecuencias que dan cuenta del fracaso de esta: el de la violencia en todos los rincones del país y la criminalización de sectores vulnerables a causa de actividades relacionadas con el cannabis. Citan que existen estudios científicos que demuestran que los efectos negativos del cannabis son menores y menos peligrosos para la salud que otras drogas autorizadas, por lo que la iniciativa propone un modelo de regulación responsable y adecuado a la realidad mexicana que opta por el cambio (Sánchez y Monreal, jueves 8 de noviembre de 2018).

Además, en la iniciativa se propone una prohibición absoluta a cualquier tipo de vínculo o actividad de menores de edad con el cannabis y se describen las facultades del IMRCC en los siguientes términos.

1) La facultad de sancionar a las personas que conduzcan vehículos bajo los niveles de THC superiores a los establecidos;

2) Brindar capacitación, asesoramiento y los insumos necesarios a los funcionarios designados para realizar los procedimientos y métodos de control;

3) Llevar el padrón anónimo del registro de plantas de cannabis para consumo personal;

4) Conceder permisos para atender más de 20 plantas para uso de salud en lo individual;

5) Crear un protocolo de investigación para el uso científico del cannabis;

6) Determinar los impuestos en la compra y venta de cannabis;

7) Establecer las disposiciones para el transporte del cannabis y sus derivados para uso terapéutico;

8) Determinar los puntos de venta del cannabis y sus derivados para fines paliativos;

9) Establecer las disposiciones para el transporte de fármacos derivados del cannabis;

10) Aprobar las características de los paquetes de cannabis y sus derivados;

11) Determinar los puntos de venta del cannabis y sus derivados para uso adulto; 
Revista Iberoamericana

de las Ciencias Sociales y

Humanísticas

ISSN: 2395 - 7972

12) Autorizar la siembra, cultivo, cosecha, preparación, fabricación, producción, distribución y venta de cannabis para fines industriales (Sánchez y Monreal, jueves 8 de noviembre de 2018).

\section{Reglamentación e impuestos de cannabis del estado de Illinois de Estados Unidos}

\section{Antecedentes}

Illinois se incorporó a la Unión Americana el 3 de diciembre de 1818 y al paso del tiempo se ha convertido en uno de los estados más prósperos de la nación estadounidense. Actualmente cuenta con una población cercana a los 13 millones de habitantes y se ha caracterizado por ser una entidad innovadora en muchos ámbitos de la vida pública, política y económica del país (Enciclopedia Libre Universal en Español, s. f.).

Como la mayoría de los estados de la Unión Americana, Illinois contaba con una legislación prohibitiva y penalizadora de la producción, consumo y comercialización de drogas y enervantes, entre ellas la de mayor consumo, el cannabis. Fue hasta que el gobernador actual, el demócrata Jay Pritzker, presentó ante la Cámara de Representantes y el Senado de Illinois una iniciativa para legalizar el consumo recreativo de mariguana para personas mayores de 21 años a partir del 1 de enero de 2020. Con 64 votos a favor y 47 en contra, dicha disposición normativa fue aprobada por el senado estatal y promulgada por el gobernador Pritzker el 25 de junio de 2019. Así, Illinois se convirtió en el onceavo estado de la nación estadounidense en legalizar el consumo recreativo de cannabis.

\section{Factores de integralidad y transversalidad de la ley}

La Ley de Reglamentación e Impuestos de Cannabis de Illinois (Illinois House Bill 1438) se constituye como todo un ejemplo de integralidad y transversalidad, ya que su contenido refleja todo un análisis, interpretación y comprensión del marco normativo y estructuras administrativas existentes en el estado de Illinois, lo que la posiciona como uno de los sistemas jurídicos más completos en el ámbito de la legalización de cannabis en Estados Unidos. 


\section{Revista Iberoamericana de las Ciencias Sociales y Humanísticas}

ISSN: $2395-7972$

Entendemos por integralidad a la incorporación y fusión de esquemas normativos y estructuras administrativas (administración pública) para el logro de un objetivo en común: poder brindar seguridad jurídica y eficiencia administrativa al proceso de legalización del consumo recreativo de cannabis. Esta fusión garantiza, entonces, una muy bien definida estrategia gubernamental para el cultivo, comercialización y consumo legal del cannabis a través del diseño e implementación de un modelo innovador en donde todos los sectores son tomados en cuenta.

En cuanto a la transversalidad, elemento fundamental para el diseño e implementación de modelos de política pública, esta ley define de manera ordenada y coherente la función que cada organismo del estado debe llevar a cabo desde el ámbito de sus respectivas competencias para que, al momento de inicio de vigencia de la ley, exista el escenario idóneo para que todos los actores que forman parte de este proceso cuenten con una adecuada delimitación de los alcances, derechos y obligaciones que esta norma jurídica les concede.

La incorporación de preceptos en la ley, relacionados con la autoridad compartida entre las diversas dependencias gubernamentales, y la definición clara respecto a temas como la equidad social en la industria de cannabis, el uso personal de cannabis, licenciamiento y regulación de organizaciones dispensadoras, centros de cultivo para adultos, organizaciones de transportación y laboratorios de prueba garantiza la transparencia y accesibilidad de las reglas para todos los sectores participantes en este proceso, lo que permite transitar paulatina pero firmemente de una etapa de penalización a otra de despenalización del consumo del cannabis con la intención de detonar los factores de desarrollo social, industrial y económico de Illinois. 
Revista Iberoamericana

de las Ciencias Sociales y

Humanísticas

ISSN: $2395-7972$

Estructura de la ley

Tabla 1. Estructura integral y transversal de la Ley de Reglamentación e Impuestos de Cannabis de Illinois

\begin{tabular}{|c|c|c|c|}
\hline Artículo & Tema & Secciones & Sumario \\
\hline Artículo 1 & $\begin{array}{l}\text { Recomendaciones y } \\
\text { definiciones }\end{array}$ & Secciones $1-1$ a $1-10$ & $\begin{array}{l}\text { Reglamento de Cannabis y } \\
\text { Ley Fiscal }\end{array}$ \\
\hline Artículo 5 & Autoridad & Secciones 5-5 a 5-45 & $\begin{array}{l}\text { Autoridad compartida: } \\
\text { - Departamento de } \\
\text { Agricultura; } \\
\text { - Departamento de } \\
\text { Regulación Financiera y } \\
\text { Profesional; } \\
\text { - Departamento de Salud } \\
\text { Pública; } \\
\text { - Departamento de } \\
\text { Servicios Humanos; } \\
\text { - Oficial de Supervisión } \\
\text { de Regulación de } \\
\text { Cannabis de Illinois. }\end{array}$ \\
\hline Artículo 7 & $\begin{array}{l}\text { Equidad social en la } \\
\text { industria de cannabis }\end{array}$ & Secciones $7-1$ a $7-30$ & $\begin{array}{l}\text { Recomendaciones: } \\
\text { - Fondo de negocios para } \\
\text { el desarrollo de } \\
\text { cannabis; } \\
\text { - Préstamos y } \\
\text { subvenciones a } \\
\text { solicitantes de equidad } \\
\text { social; } \\
\text { - Exenciones de cuotas; } \\
\text { - Transferencia de licencia } \\
\text { otorgada a solicitante de } \\
\text { equidad social; } \\
\text { - Informes. }\end{array}$ \\
\hline $\begin{array}{l}\text { Artículo } \\
10\end{array}$ & $\begin{array}{l}\text { Uso personal de } \\
\text { cannabis }\end{array}$ & $\begin{array}{l}\text { Secciones } 10-5 \text { a } 10- \\
50\end{array}$ & $\begin{array}{l}\text { - Uso personal de } \\
\text { cannabis, restricciones } \\
\text { en el cultivo y multas: } \\
\text { - Personas menores de } 21 \\
\text { años; } \\
\text { - Identificación, falsa } \\
\text { identificación y multas; } \\
\text { - Inmunidades y } \\
\text { presunciones }\end{array}$ \\
\hline
\end{tabular}


Revista Iberoamericana

de las Ciencias Sociales y

Humanísticas

ISSN: $2395-7972$

\begin{tabular}{|c|c|c|c|}
\hline & & & $\begin{array}{l}\text { relacionadas por compra } \\
\text { de cannabis, para su uso; } \\
\text { - Prohibición de la } \\
\text { discriminación } \\
\text { - Limitaciones y multas; } \\
\text { - Restaurar, reinvertir y } \\
\text { renovar el programa; } \\
\text { - Empleo y } \\
\text { responsabilidad del } \\
\text { empleador. }\end{array}$ \\
\hline $\begin{array}{l}\text { Artículo } \\
15\end{array}$ & $\begin{array}{l}\text { Licencias y } \\
\text { regulación de } \\
\text { organizaciones } \\
\text { dispensadoras }\end{array}$ & $\begin{array}{l}\text { Secciones } 15-5 \text { a } 15- \\
175\end{array}$ & $\begin{array}{l}\text { Autoridad: } \\
\text { - Exención de la } \\
\text { organización } \\
\text { dispensadora de } \\
\text { cannabis medicinal; } \\
\text { - Aprobación temprana de } \\
\text { licencia de organización } \\
\text { dispensadora de uso de } \\
\text { adultos; } \\
\text { - Responsabilidad } \\
\text { financiera; } \\
\text { - Administración; } \\
\text { - Agentes a cargo; } \\
\text { - Requerimientos de } \\
\text { almacenaje; } \\
\text { - Requerimientos de } \\
\text { operación; } \\
\text { - Seguridad; } \\
\text { - Destrucción y } \\
\text { disponibilidad de } \\
\text { cannabis; } \\
\text { - Investigación; } \\
\text { - A partir del } 1 \text { de enero } \\
\text { de 2022 el } \\
\text { Departamento Licencias } \\
\text { deberá modificar } \\
\text { cualquier tarifa } \\
\text { establecida en este } \\
\text { artículo. } \\
\end{array}$ \\
\hline $\begin{array}{l}\text { Artículo } \\
20\end{array}$ & $\begin{array}{l}\text { Centros de cultivo } \\
\text { para adultos }\end{array}$ & $\begin{array}{l}\text { Secciones 20-1 a 20- } \\
50\end{array}$ & $\begin{array}{l}\text { - Emisión de licencias. A } \\
\text { partir del } 1 \text { de julio de } \\
\text { 2021, el Departamento } \\
\text { de Agricultura podrá } \\
\text { cambiar el número de } \\
\text { licencias para centros de } \\
\text { cultivo. } \\
\end{array}$ \\
\hline
\end{tabular}


Revista Iberoamericana

de las Ciencias Sociales y

Humanísticas

ISSN: 2395 - 7972

\begin{tabular}{|c|c|c|c|}
\hline $\begin{array}{l}\text { Artículo } \\
25\end{array}$ & $\begin{array}{l}\text { Programa piloto } \\
\text { vocacional de } \\
\text { cannabis de la } \\
\text { universidad } \\
\text { comunitaria }\end{array}$ & $\begin{array}{l}\text { Secciones } 25-1 \text { a } 25- \\
20\end{array}$ & $\begin{array}{l}\text { Definiciones: } \\
\text { - Administración; } \\
\text { - Emisión de licencias de } \\
\text { programas piloto } \\
\text { vocacionales de } \\
\text { cannabis en colegios } \\
\text { comunitarios; } \\
\text { - Programa pilotos } \\
\text { vocacionales de } \\
\text { cannabis en colegios } \\
\text { comunitarios: } \\
\text { requerimientos y } \\
\text { prohibiciones. }\end{array}$ \\
\hline $\begin{array}{l}\text { Artículo } \\
30\end{array}$ & Arte de cultivo & Sección 30-3 a 30-50 & $\begin{array}{l}\text { Emisión de licencias: } \\
\text { - Solicitud; } \\
\text { - Aplicación de } \\
\text { puntuación; } \\
\text { - Denegación de solicitud; } \\
\text { - Requisitos para } \\
\quad \text { productor artesanal. }\end{array}$ \\
\hline $\begin{array}{l}\text { Artículo } \\
35\end{array}$ & $\begin{array}{l}\text { Organizaciones de } \\
\text { infusión }\end{array}$ & Sección 35-3 a 35-40 & $\begin{array}{l}\text { Definiciones: } \\
\text { - Emisión de licencias; } \\
\text { - Solicitud; } \\
\text { - Denegación de } \\
\text { solicitudes; } \\
\text { - Tarjeta de identificación } \\
\text { de agente. }\end{array}$ \\
\hline $\begin{array}{l}\text { Artículo } \\
40\end{array}$ & $\begin{array}{l}\text { Organización para } \\
\text { transportación }\end{array}$ & $\begin{array}{l}\text { Secciones 40-1 a 40- } \\
35\end{array}$ & $\begin{array}{l}\text { Definiciones: } \\
\text { - Emisión de licencias; } \\
\text { - Solicitud; } \\
\text { - Emitiendo licencias; } \\
\text { - Denegación de } \\
\text { solicitudes; } \\
\text { - Requisitos de la } \\
\text { organización de } \\
\text { transporte; } \\
\text { - Tarjeta de identificación } \\
\text { del agente de } \\
\text { transportación; } \\
\text { - Verificación de } \\
\text { antecedentes de la } \\
\text { organización de } \\
\text { transporte. }\end{array}$ \\
\hline $\begin{array}{l}\text { Artículo } \\
45\end{array}$ & $\begin{array}{l}\text { Aplicación e } \\
\text { inmunidades }\end{array}$ & $\begin{array}{l}\text { Secciones } 45-5 \text { a } 45- \\
20\end{array}$ & $\begin{array}{l}\text { - Suspensión de licencias, } \\
\text { revocación y otras } \\
\text { multas; }\end{array}$ \\
\hline
\end{tabular}


Revista Iberoamericana

de las Ciencias Sociales y

Humanísticas

ISSN: 2395 - 7972

\begin{tabular}{|c|c|c|c|}
\hline & & & $\begin{array}{l}\text { - Inmunidades y } \\
\text { presunciones } \\
\text { relacionadas con el } \\
\text { manejo de cannabis por } \\
\text { establecimientos } \\
\text { comerciales de cannabis } \\
\text { y sus agentes; } \\
\text { - Normas estatales y } \\
\text { requisitos; } \\
\text { - Violación de las leyes } \\
\text { fiscales; rechazo, } \\
\text { revocación o suspensión } \\
\text { de la licencia o tarjeta de } \\
\text { identificación del } \\
\text { agente. }\end{array}$ \\
\hline $\begin{array}{l}\text { Artículo } \\
50\end{array}$ & $\begin{array}{l}\text { Pruebas de } \\
\text { laboratorio }\end{array}$ & Sección 50-5 & Única. \\
\hline $\begin{array}{l}\text { Artículo } \\
55\end{array}$ & $\begin{array}{l}\text { Disposiciones } \\
\text { generales }\end{array}$ & $\begin{array}{l}\text { Secciones 55-5 a 55- } \\
95\end{array}$ & $\begin{array}{l}\text { - Preparación de } \\
\text { productos con infusión } \\
\text { de cannabis; } \\
\text { - Mantenimiento de } \\
\text { inventario; } \\
\text { - Destrucción de cannabis } \\
\text { - Publicidad y promoción. }\end{array}$ \\
\hline $\begin{array}{l}\text { Artículo } \\
60\end{array}$ & $\begin{array}{l}\text { Impuesto de } \\
\text { privilegio de cultivo } \\
\text { de cannabis }\end{array}$ & $\begin{array}{l}\text { Secciones } 60-1 \text { a } 60- \\
45\end{array}$ & $\begin{array}{l}\text { Este artículo puede ser } \\
\text { referido } \\
\text { como Ley de Impuestos de } \\
\text { Privilegio de Cultivo de } \\
\text { Cannabis: } \\
\text { - Impuesto; } \\
\text { - Registro de cultivadores; } \\
\text { - Devolución y pago del } \\
\text { impuesto por cultivo de } \\
\text { cannabis. }\end{array}$ \\
\hline $\begin{array}{l}\text { Artículo } \\
65\end{array}$ & $\begin{array}{l}\text { Impuesto especial al } \\
\text { comprador de } \\
\text { cannabis }\end{array}$ & $\begin{array}{l}\text { Secciones } 65-1 \text { a } 65- \\
50\end{array}$ & $\begin{array}{l}\text { Este artículo puede ser } \\
\text { referido } \\
\text { como Ley de Impuestos } \\
\text { Especiales del Comprador } \\
\text { de Cannabis. }\end{array}$ \\
\hline $\begin{array}{l}\text { Artículo } \\
900\end{array}$ & $\begin{array}{l}\text { Disposiciones y } \\
\text { enmiendas }\end{array}$ & $\begin{array}{l}\text { Secciones } 900-5 \text { a } \\
900-50\end{array}$ & $\begin{array}{l}\text { La Ley de Procedimiento } \\
\text { Administrativo de Illinois. } \\
\text { Se modifican diversos } \\
\text { artículos y secciones. }\end{array}$ \\
\hline $\begin{array}{l}\text { Artículo } \\
999\end{array}$ & $\begin{array}{l}\text { Disposiciones y } \\
\text { misceláneas }\end{array}$ & $\begin{array}{l}\text { Secciones 999-95 y } \\
999-99\end{array}$ & $\begin{array}{l}\text { Sin aceleración o retraso: } \\
\text { Donde esta ley realiza } \\
\text { cambios en un estatuto que } \\
\text { está representado en esta }\end{array}$ \\
\hline
\end{tabular}




\begin{tabular}{|l|l|l|}
\hline & $\begin{array}{l}\text { por texto que aún no está } \\
\text { vigente o ya no está vigente } \\
\text { (por ejemplo, una } \\
\text { sección representada por } \\
\text { varias versiones), el uso de } \\
\text { ese texto no acelera ni } \\
\text { retrasa los efectos de los } \\
\text { cambios realizados por esta } \\
\text { Ley o disposiciones } \\
\text { derivadas de cualquier otra } \\
\text { ley pública. }\end{array}$ \\
\hline
\end{tabular}

Fuente: Elaboración propia con base en Illinois General Assembly (2019a)

\section{Puntos relevantes de la ley}

Del análisis de los resultados de la tabla 1, se determinó que la policía se concentra en delitos violentos y de propiedad que repercuten en la reducción de ingresos destinados a temas como la educación, prevención y tratamiento del abuso de sustancias, por lo que a partir de esta iniciativa se liberarán recursos para invertir en comunidades y otros fines públicos, motivo por el cual se legaliza el consumo de cannabis en el estado de Illinois (Illinois General Assembly, 2019a). Además, según la enmienda que modifica el proyecto de ley 1438 de la Cámara (Illinois General Assembly, 2019b):

a) El cannabis se regulará de forma similar al del alcohol;

b) Consumos solo mayores de 21 años;

c) Pago de impuestos similares a los del alcohol;

d) Vender, transferir o distribuir cannabis a menores de edad permanece ilegal;

e) Borrado de antecedentes penales por delitos menores de esta índole;

f) El cannabis para uso recreativo se podrá adquirir en dispensarios certificados;

g) La ley deja de sancionar con prisión a los infractores, por lo tanto, aquellos que cultiven sin permiso solo recibirán una multa de 200 dólares;

h) De los ingresos fiscales generados por la comercialización del cannabis, el $35 \%$ se destinará al Fondo General del Estado;

i) Queda prohibido por la ley la publicidad sobre consumo de cannabis cerca de escuelas, patios de recreo, transporte público y cualquier tipo de publicidad destinada a menores de edad. 


\section{Los objetivos y atribuciones del IMRCC}

Los objetivos del IMRCC deben estar dirigidos a garantizar el enfoque de salud pública, para sanar la problemática del fenómeno analizado, las actividades del sistema, las medidas de seguridad, de vigilancia, las acciones para reducir los riesgos y daños asociados al uso del cannabis, evaluar la regulación que se establezca y difundir información sobre el sistema.

1) Deberá crear la regulación que garantice el enfoque de salud pública, de reducción de riesgos y daños relacionados con el consumo de cannabis;

2) Deberá reglamentar la plantación, el cultivo, la cosecha, el transporte, el almacenaje, la producción, elaboración, distribución, comercialización, expendio y venta del cannabis;

3) Aplicará medidas de seguridad y sanciones por el aseguramiento de productos que sean nocivos o carezcan de los requisitos básicos;

4) Valorar la regulación en los usos del cannabis.

De igual forma, se deben establecer las atribuciones que le competan al IMRCC para establecer los lineamientos de las licencias, otorgar licencias y prórrogas para fines personales, medicinales y comerciales, implementar medidas afirmativas, aplicar sanciones administrativas, realizar investigación científica, establecer el registro anónimo de autoproductores, crear el padrón de cooperativas, autorizar la importación y exportación del cannabis, controlar la información estadística y personal y expedir su estatuto orgánico.

\section{Resultados}

A continuación, se citan algunos datos de la Encuesta Nacional de Consumo de Drogas, Alcohol y Tabaco (Encodat) 2016-2017 (Comisión Nacional contra las Adicciones, 2017). Consumo de drogas: prevalencias globales, tendencias y variaciones estatales.

Resultados principales. Datos:

1) Población total (12-65 años):

a) $\quad 10.3 \%$ ha consumido cualquier droga alguna vez en la vida; $2.9 \%$ lo hizo en el último año (2.5 millones) y $1.5 \%$ en el último mes.

b) $\quad 9.9 \%$ ha consumido drogas ilegales alguna vez en la vida (15.8\% hombres y $4.3 \%$ mujeres); $2.7 \%$ las ha consumido en el último año (4.4\% 
Revista Iberoamericana

de las Ciencias Sociales y

Humanísticas

ISSN: $2395-7972$

hombres y $1.1 \%$ mujeres) y $1.4 \%$ en el último mes $(2.6 \%$ hombres, $0.4 \%$ mujeres).

c) $\quad 8.6 \%$ ha consumido mariguana alguna vez en la vida, $2.1 \%$ en el último año (1.8 millones) y $1.2 \%$ en el último mes.

d) La edad de inicio de consumo de drogas es de 17.8 años (hombres 17.7 y mujeres 18.2).

2) Población adolescente (12-17 años):

a) $\quad 6.4 \%$ ha consumido cualquier droga alguna vez, $3.1 \%$ lo ha hecho en el último año (437 000) y $1.2 \%$ en el último mes.

b) $\quad 6.2 \%$ ha consumido drogas ilegales alguna vez (6.6\% hombres y $5.8 \%$ mujeres), $2.9 \%$ en el último año (3.4\% hombres y $2.3 \%$ mujeres) y $1.2 \%$ lo ha hecho en el último mes ( $1.7 \%$ hombres, $0.7 \%$ mujeres).

c) $\quad 5.3 \%$ ha consumido mariguana alguna vez; en el último año $2.6 \%$ ha consumido mariguana (373 000); en el último mes, $1.1 \%$ ha consumido mariguana.

3) Población adulta (18-65 años):

a) En la población de 18 a 34 años (en este grupo se encuentra la mayor prevalencia global de consumo):

i. $15 \%$ ha consumido cualquier droga alguna vez en la vida $(22.8 \%$ hombres, $7.6 \%$ mujeres).

ii. $5 \%$ ha consumido cualquier droga en el último año (8.2\% hombres, $2.0 \%$ mujeres).

iii. $2.7 \%$ ha consumido cualquier droga en el último mes (4.8\% hombres y $0.7 \%$ mujeres).

iv. $14.5 \%$ ha consumido drogas ilegales alguna vez $(22.6 \%$ hombres y $7 \%$ mujeres).

v. $4.6 \%$ ha consumido drogas ilegales en el último año $(7.8 \%$ hombres y $1.6 \%$ mujeres).

vi. $2.5 \%$ ha consumido drogas ilegales en el último mes (4.6\% hombres, $0.6 \%$ mujeres).

vii. La mariguana y la cocaína son las drogas de mayor preferencia (12.8\% y $5.2 \%$ respectivamente para alguna vez, $3.5 \%$ y $1.5 \%$ en 
Revista Iberoamericana

de las Ciencias Sociales y Humanísticas

ISSN: $2395-7972$

el último año y $2 \%$ y $0.8 \%$ respectivamente para último mes).

b) En la población de 35 a 65 años:

i. La prevalencia de consumo alguna vez de cualquier droga es de $7.5 \%$ (13.8\% hombres y $1.8 \%$ mujeres $)$, mientras que la de drogas ilegales es de $7 \%$ (13.4\% hombres y $1.3 \%$ mujeres).

ii. La prevalencia de cualquier droga en el último año es de $1 \%$ (1.8\% hombres y $0.3 \%$ mujeres) y la de drogas ilegales es de $0.8 \%$ (1.6\% hombres y $0.1 \%$ mujeres).

iii. En el último mes, la prevalencia de consumo de cualquier droga es de $0.6 \%$ (1.2\% hombres y $0.2 \%$ mujeres) y la de drogas ilegales es de $0.5 \%$ ( $1 \%$ hombres y $<0.1 \%$ mujeres $)$.

Tendencias en el consumo:

1) Población total (12-65 años):

a) La prevalencia de cualquier droga alguna vez aumentó de $7.8 \%$ en 2011 a $10.3 \%$ en 2016.

b) La prevalencia de drogas ilegales alguna vez aumentó de $7.2 \%$ a $9.9 \%$.

c) La prevalencia alguna vez de mariguana pasó de $6 \%$ a $8.6 \%$.

2) Población adolescente (12-17 años):

a) La prevalencia alguna vez de drogas ilegales aumentó significativamente de $2.9 \%$ a $6.2 \%$.

b) La mariguana aumentó significativamente tanto en el consumo alguna vez de $2.4 \%$ a $5.3 \%$.

3) Población adulta (18-65 años):

a) En la población de 18 a 34 años:

i. La prevalencia de consumo de cualquier droga alguna vez incrementó de $11.3 \%$ en 2011 a $15 \%$ en 2016

b) En la población de 35 a 65 años:

i. El consumo de cualquier droga alguna vez pasó de $6.4 \%$ a $7.5 \%$ y el consumo de drogas ilegales alguna vez pasó de $5.7 \%$ a $7 \%$ de 2011 a 2016.

4) Variaciones estatales: 
Revista Iberoamericana

de las Ciencias Sociales y

Humanísticas

ISSN: 2395 - 7972

a) Jalisco (15.3\%), Quintana Roo (14.9\%) y Baja California (13.5\%) reportan los porcentajes más altos de consumo de cualquier droga ilegal alguna vez en la población de 12 a 65 años, con respecto a la prevalencia nacional $(9.9 \%)$.

b) En el último año, solo Baja California (4.4\%) tiene un porcentaje mayor al nacional $(2.7 \%)$.

Con los datos estadísticos que se citan se demuestra la tendencia en aumento al consumo del cannabis, por lo que se puede afirmar que es un fenómeno social ineludible para la ciencia. Además, llama la atención el consumo de los menores de edad de este psicotrópico, por lo que se sostiene que la política prohibicionista existente en México no ha atendido de forma positiva el fenómeno en análisis, pues es un problema de salud pública; el hecho de que los menores de edad lo consuman, en consecuencia, la propuesta de la nueva política de regulación debe ser con una prohibición absoluta para ellos, es decir, los menores de edad.

\section{Discusión}

Ha servido de guía el atrayente libro de Serra (2013): en uno de los apartados sostiene que la administración pública paraestatal está integrada por el conjunto de instituciones, organismos, empresas de economía mixta, patrimonios públicos que por disposición de la ley colaboran en la relación de los fines del Estado. Y desde luego la sugerente afirmación de Sánchez y Monreal (8 de noviembre de 2018) en la que sostienen que entre las facultades del IMRCC deberá contar con una sancionadora, de capacitación, de proveedor, registral, de cesión de permisos, de investigación científica, de determinación de tributos; de lo anterior se observa que el ente garante en estudio, sea de la administración pública centralizada o pertenezca a la descentralizada (una vez que se apruebe la ley se corroborará), deberá contar necesariamente con las facultades mencionadas. Lo anterior, se analizó utilizándolo como hilo conductor, sin embargo, no escapa a esta investigación lo difícil de acceder a fuentes de primera mano (como informantes claves) por ser un tema con muchos tabúes o por la política prohibitiva hasta la fecha existente, considerando como una de sus fortalezas lo novedoso que será en México. Asimismo, el poder poner como objeto de estudio la norma jurídica que regule, ya sin limitación, para la investigación científica. 


\section{Conclusiones}

El ente garante de la regulación jurídica del cannabis en México debe ser un organismo descentralizado de la administración pública federal, integrado por miembros de la Secretaría de Salud, de la Cofepris y de la Secretaría de Gobernación, que forme parte de la administración pública paraestatal con personalidad jurídica y patrimonio propio, además de dotarse de autonomía de gestión. Se encargará de regular, reglamentar, monitorear, sancionar y evaluar el sistema de regulación del cannabis; su origen constitucional se encuentra en el artículo 90 de la Constitución mexicana y su fuente secundaria está en los artículos 3 y 17 de la Ley Orgánica de la Administración Pública Federal (DOF, 29 de diciembre de 1976), por lo que debe crear para su funcionamiento interno sus propios ordenamientos.

El IMRCC requerirá que se le dote de facultades fortalecidas en la ley y basadas en los principios constitucionales, para poder operar y hacer realidad el derecho fundamental que debe cristalizar. Entre esas facultades deberá contar con una facultad sancionadora, de capacitación, de proveedor, registral, de cesión de permisos, de investigación científica, de determinación de tributos, establecer las destrezas para el transporte, los puntos de venta, las características de embalaje, así como autorizar el funcionamiento de cada una de las fases que integrarán el sistema; todo ello haciendo énfasis en la política prohibicionista absoluta a cualquier tipo de vínculo o actividad de los menores de edad con el cannabis.

En aras de un comparativo internacional, se analizó la Ley de Reglamentación e Impuestos de Cannabis del estado de Illinois de Estados Unidos, la cual contiene similitudes y diferencias respecto a la propuesta de ley en México. Entre las primeras se encontró su estructura administrativa en comparación con el IMRCC, que será un organismo descentralizado. Respecto a las segundas, destaca que es una ley fiscal al establecer impuestos sobre el sistema del cannabis; en México el IMRCC solo determinará el pago de derechos, pues el establecimiento de impuestos está reservado constitucionalmente a la Cámara de Diputados del Congreso de la Unión.

De la estadística citada se fundamenta el por qué o la justificación de investigación sobre este fenómeno, pues de ahí se desprende la tendencia en aumento del consumo de este psicotrópico, más aún por menores de edad. Así pues, se afirma que la política prohibicionista actualmente vigente en México no ha dado respuesta positiva a este fenómeno social, por lo que se debe avanzar hacia una nueva regulación jurídica del cannabis en México que dé 
Revista Iberoamericana

de las Ciencias Sociales y

Humanísticas

ISSN: $2395-7972$

origen al ente garante para cristalizar el derecho fundamental al libre desarrollo de la personalidad de todos los individuos mexicanos.

\section{Referencias}

Cámara de Diputados del H. Congreso de la Unión. (1917). Constitución Política de los Estados Unidos Mexicanos, 1917. México: Cámara de Diputados del H. Congreso de la Unión. Recuperado de http://www.diputados.gob.mx/LeyesBiblio/htm/1.htm.

Cámara de Diputados del H. Congreso de la Unión. (29 de diciembre de 1976). Ley Orgánica de la Administración Pública Federal. Diario Oficial de la Federación. Recuperado de

https://www.gob.mx/cms/uploads/attachment/file/487996/Ley_Org_nica_APF_0908-19.pdf.

Cámara de Diputados del H. Congreso de la Unión. (26 de enero de 1990). Ley Federal de las Entidades Paraestatales. Diario Oficial de la Federación. Recuperado de http://www.diputados.gob.mx/LeyesBiblio/regley/Reg_LFEP.pdf.

Comisión Nacional contra las Adicciones. (2017). Encuesta Nacional de Consumo de

Drogas, Alcohol y Tabaco (Encodat) 2016-2017. México: Comisión Nacional contra las Adicciones. Recuperado de https://www.gob.mx/salud\%7Cconadic/acciones-yprogramas/encuesta-nacional-de-consumo-de-drogas-alcohol-y-tabaco-encodat2016-2017-136758.

Couto, V. (2014). La legalización de la marihuana en México. (tesis de maestría). Instituto Tecnologico y de Estudios Superiores de Monterrey, Ciudad de México. Recuperado de https://repositorio.tec.mx/bitstream/handle/11285/630109/33068001114298.pdf?seq uence $=1 \&$ is Allowed $=\mathrm{y}$.

de Pina García, J. P. y de Pina Vara, R. y de Pina, R. (2012). Diccionario de Derecho (37. ed.). México: Porrúa.

Enciclopedia Libre Universal en Español. (s. f.) Illinois. Recuperado de http://enciclopedia.us.es/index.php/Illinois_(Estados_Unidos_de_Am\%C3\%A9rica) Hamdan, F. (2016). Derecho administrativo. México: SCJN y ELD. 
Revista Iberoamericana

de las Ciencias Sociales y

Humanísticas

ISSN: $2395-7972$

Illinois General Assembly. (2019a). Public Act 101-0027. Chicago, United States: Illinois

General

Assembly.

Retrieved

from

http://www.ilga.gov/legislation/BillStatus.asp?DocNum=1438\&GAID=15\&DocTy peID=HB\&SessionID $=108 \& G A=101$.

Illinois General Assembly. (2019b). Amendment to House Bill 1438. Chicago, United States: Illinois General Assembly. Retrieved from http://www.ilga.gov/legislation/101/HB/PDF/10100HB1438sam002.pdf.

Organización de los Estados Americanos [OEA]. (2019). Informe sobre el consumo de drogas en las américas 2019. Washington, Estados Unidos: Organización de los Estados Americanos.

Sánchez, O. y Monreal, R. (8 de noviembre de 2018). De la Sen. Olga Sánchez Cordero Dávila y del Sen. Ricardo Monreal Ávila, con aval del Grupo Parlamentario Morena, con proyecto de decreto por el que se expide la Ley General para la Regulación y Control de Cannabis. Gaceta del Senado. Recuperado de https://www.senado.gob.mx/64/gaceta_del_senado/documento/85686.

Segunda Sala de la Suprema Corte de Justicia de la Nación. (2013). Organismos descentralizados y presidente de la república. Entre ellos no existe una relación de jerarquía directa. México: Suprema Corte de Justicia de la Nación. Recuperado de http://sjf.scjn.gob.mx/SJFSist/Paginas/DetalleGeneralV2.aspx?ID=2003677\&Clase $=$ DetalleTesisBL $\&$ Semanario $=0$.

Serra, A. (2013). Derecho administrativo (29. a ed.). México: Porrúa. 
Revista Iberoamericana

de las Ciencias Sociales y

Humanísticas

\begin{tabular}{|c|c|}
\hline Rol de Contribución & Autor (es) \\
\hline Conceptualización & Alejandro Sánchez Sánchez \\
\hline Metodología & Alejandro Sánchez Sánchez \\
\hline Software & Luis Carlos Castro Vizcarra \\
\hline Validación & Alejandro Sánchez Sánchez \\
\hline Análisis Formal & Alejandro Moctezuma Hernández. \\
\hline Investigación & Gloria Aurora de Las Fuentes Lacavex \\
\hline Recursos & Sergio Cruz Hernández \\
\hline Curación de datos & Luis Carlos Castro Vizcarra \\
\hline $\begin{array}{l}\text { Escritura - Preparación del } \\
\text { borrador original }\end{array}$ & Alejandro Sánchez Sánchez \\
\hline $\begin{array}{l}\text { Escritura - Revisión y } \\
\text { edición }\end{array}$ & Luis Enrique Concepción Montiel. \\
\hline Visualización & Gloria Aurora De Las Fuentes Lacavex \\
\hline Supervisión & Alejandro Sánchez Sánchez \\
\hline $\begin{array}{l}\text { Administración de } \\
\text { Proyectos }\end{array}$ & Alejandro Sánchez Sánchez \\
\hline Adquisición de fondos & Sergio Cruz Hernández. \\
\hline
\end{tabular}

